\title{
A functional SNP associated with atopic dermatitis controls cell type-specific methylation of the VSTM1 gene locus
}

Dilip Kumar ${ }^{1 \dagger}$, Kia Joo Puan ${ }^{1 \dagger}$, Anand Kumar Andiappan ${ }^{1 \dagger}$, Bernett Lee ${ }^{1}$, Geertje H. A. Westerlaken², Doreen Haase ${ }^{1}$, Rossella Melchiotti ${ }^{1}$, Zhuang Li ${ }^{1}$, Nurhashikin Yusof ${ }^{1}$, Josephine Lum¹', Geraldine Koh'1, Shihui Foo ${ }^{1}$, Joe Yeong ${ }^{1,16}$, Alexessander Couto Alves ${ }^{3}$, Juha Pekkanen ${ }^{4}$, Liang Dan Sun ${ }^{5}$, Astrid Irwanto ${ }^{6}$, Benjamin P. Fairfax ${ }^{7,8}$,

Vivek Naranbhai ${ }^{7,8}$, John E. A. Common ${ }^{9}$, Mark Tang ${ }^{12}$, Chin Keh Chuang ${ }^{17,18}$, Marjo-Riitta Jarvelin 3,13,14,15, Julian C. Knight ${ }^{7}$, Xuejun Zhang ${ }^{5}$, Fook Tim Chew ${ }^{19}$, Shyam Prabhakar ${ }^{6}$, Liu Jianjun', De Yun Wang ${ }^{10,11}$, Francesca Zolezzi ${ }^{1}$, Michael Poidinger ${ }^{1}$, E. Birgitte Lane ${ }^{9}$, Linde Meyaard ${ }^{2^{* \dagger}}$ and Olaf Rötzschke ${ }^{1^{* \dagger}}$

\begin{abstract}
Background: Expression quantitative trait loci (eQTL) databases represent a valuable resource to link diseaseassociated SNPs to specific candidate genes whose gene expression is significantly modulated by the SNP under investigation. We previously identified signal inhibitory receptor on leukocytes-1 (SIRL-1) as a powerful regulator of human innate immune cell function. While it is constitutively high expressed on neutrophils, on monocytes the SIRL-1 surface expression varies strongly between individuals. The underlying mechanism of regulation, its genetic control as well as potential clinical implications had not been explored yet.

Methods: Whole blood eQTL data of a Chinese cohort was used to identify SNPs regulating the expression of VSTM1, the gene encoding SIRL-1. The genotype effect was validated by flow cytometry (cell surface expression), correlated with electrophoretic mobility shift assay (EMSA), chromatin immunoprecipitation (ChIP) and bisulfite sequencing (C-methylation) and its functional impact studied the inhibition of reactive oxygen species (ROS).

Results: We found a significant association of a single CpG-SNP, rs612529T/C, located in the promoter of VSTM1. Through flow cytometry analysis we confirmed that primarily in the monocytes the protein level of SIRL-1 is strongly associated with genotype of this SNP. In monocytes, the T allele of this SNP facilitates binding of the transcription factors YY1 and PU.1, of which the latter has been recently shown to act as docking site for modifiers of DNA methylation. In line with this notion rs612529T associates with a complete demethylation of the VSTM1 promoter correlating with the allele-specific upregulation of SIRL-1 expression. In monocytes, this upregulation strongly impacts the IgA-induced production of ROS by these cells. Through targeted association analysis we found a significant Meta $P$ value of $1.14 \times 10^{-6}$ for rs612529 for association to atopic dermatitis (AD).

(Continued on next page)
\end{abstract}

\footnotetext{
* Correspondence: L.Meyaard@umcutrecht.nl;

Olaf_Rotzschke@immunol.a-star.edu.sg

${ }^{\dagger}$ Equal contributors

2Laboratory of Translational Immunology, Department of Immunology, University Medical Center Utrecht, P.O. box 85090, Utrecht 3508 AB, The Netherlands

${ }^{1}$ Singapore Immunology Network (SIgN), A*STAR (Agency for Science,

Technology and Research), 8A Biomedical Grove \#04-06, Singapore 138648,

Republic of Singapore

Full list of author information is available at the end of the article
} 
(Continued from previous page)

Conclusion: Low expression of SIRL-1 on monocytes is associated with an increased risk for the manifestation of an inflammatory skin disease. It thus underlines the role of both the cell subset and this inhibitory immune receptor in maintaining immune homeostasis in the skin. Notably, the genetic regulation is achieved by a single CpG-SNP, which controls the overall methylation state of the promoter gene segment.

Keywords: VSTM1, Signal inhibitory receptor on leukocytes-1 (SIRL-1), Expression quantitative trait loci (eQTL), Atopic dermatitis, Monocytes, Reactive oxygen species (ROS), Neutrophils

\section{Background}

Genome-wide association studies (GWAS) in the recent past have identified thousands of disease-related genetic variants and trait-associated polymorphisms [1-3]. The National Genome Research Institute (NHGRI) GWAS catalog alone comprises data of more than 2000 different studies organized with association $P$ values from hundreds of disease phenotypes and complex traits [4]. However, translating these associated variants to causality and potential mechanisms of disease have been difficult. Recent advances in high throughput genotyping and gene expression analysis have allowed for an extensive mapping of expressed quantitative trait loci (eQTL) [5, 6]. Although many of these eQTL are cell-typespecific and also vary between the ethnic groups, they represent a valuable resource for candidate proteins, whose messenger RNA (mRNA) expression is, at least in part, genetically controlled [5-7]. These eQTL datasets provide an excellent opportunity to address and understand the mechanisms underlying GWAS hits. Zhu et al. have recently demonstrated this at a whole genome level by providing a list of priority candidate genes for further functional evaluation of the five complex human traits [8]. However, once the priority candidate genes have been identified, it needs to be validated using well designed in vitro, in vivo, or ex vivo models using techniques, which go beyond assaying the genome and transcriptome $[9,10]$. Additionally, this also requires knowledge on the protein molecule under investigation or pathways identified, cell/tissue specificity, and functional consequence on the immune system. Hence, there is a need for targeted but yet comprehensive candidate gene-based studies looking at various aspects of the potential disease gene from DNA to protein, also in pure populations of immune cells and/or tissues with appropriate stimuli and functional readouts. In this study, we present an in-depth, well-characterized study of the SIRL-1 (signal inhibitory receptor on leukocytes-1) gene using various complementary techniques.

The "immune inhibitory receptors" represent a heterogeneous family of transmembrane receptors that function as negative regulators of immune cells [11, 12]. Upon ligation, the inhibitory signal is transduced through immunoreceptor tyrosine-based inhibitory motifs (ITIMs) located in the cytoplasmic tail of the receptor [13]. One member of this family is SIRL-1. While the natural ligand is not known yet, cross-linking of the receptor with agonistic antibodies results in the tyrosine phosphorylation of the ITIMs by the Src family kinases (SFK). The phosphorylated ITIMs act as a docking site for the recruitment of the Src homology 2 (SH2) domain-containing tyrosine phosphatases SHP-1 and/or SHP-2[14]. These phosphatases antagonize the effects of activating SFK and Syk family kinases, triggered for instance by the engagement of IgE-specific Fc-epsilon receptors [15]. Therefore, these immune inhibitory receptors have been implicated to play a role in regulating antibody-mediated immune responses. SIRL-1 is known to primarily express on human myeloid cells, where it inhibits crucial pro-inflammatory functions, such as $\mathrm{Fc}$ receptor $(\mathrm{FcR})$ mediated respiratory burst and neutrophil extracellular trap (NET) formation [12, 14, $16,17]$. In a prior study, we made an observation reporting striking differences in the expression level of SIRL-1 on various immune subsets. Granulocyte subsets neutrophils and eosinophils constitutively expressed high levels SIRL-1 on most individuals, while the expression level of the receptor on monocytes varied strongly between individuals [16]. The cause of these variations as well as the potential clinical implications was not known.

In this study, we used mRNA and genotype data from two independent cohorts (Chinese, Caucasian) to link population variations of the molecule on monocytes to a single single nucleotide polymorphism (SNP), rs612529T/C, a CpG-SNP located in the promoter region of the SIRL-1 encoding gene VSTM1. By employing flow cytometry (FACS), functional assays, electrophoretic mobility shift assay (EMSA), bisulfite DNA sequencing, and chromatin immune precipitation (ChIP) analysis, we could further show that SIRL-1 upregulation correlates with the allele dependent binding of PU.1 and YY1 to the CpG-SNP, which in turn is associated with a demethylation of the entire promoter region. Importantly, genetic association analysis suggested that the cell-type-specific fluctuations of SIRL-1 indeed might have pathologic consequences. We observed that in three independent cohorts, the $\mathrm{C}$ allele (indicative of low expression) is consistently associated with an increased risk for atopic dermatitis $(\mathrm{AD})$, an inflammatory skin condition. 


\section{Methods}

\section{Human blood samples and cohorts}

The cohorts used in this study were outlined in Additional file 1. eQTL analysis was carried out with ethnic Chinese cohorts collected in Singapore ("blood eQTL cohort" and "monocyte \& B cell eQTL cohort") of 202 (unpublished) and 15 individuals [18], respectively. In addition, data were used from a "Monocyte \& B cell cohort" of 279 individuals of Caucasian ethnicity that had been reported before by Fairfax et al. [19] With the exception of the "functional cohort" (see below), details on all cohorts had been published previously. Data of three additional cohorts (Singapore Chinese population [20], Chinese Han Population [21] and Caucasian population [22]) that had been published previously were used for the genetic association of the VSTM1-SNP rs612529 with AD.

\section{Functional cohort}

Another genotyped Singaporean cohort consisting of ethnic Chinese individuals was used for the functional characterization of VSTM1 by FACS $(n=44)$, reactive oxygen species (ROS) assay $(\mathrm{n}=30)$, DNA-methylation $(\mathrm{n}=8)$, and ChIP assay $(\mathrm{n}=9)$ ("functional cohort"). For all individuals of the "functional cohort," the rs612529T/ $\mathrm{C}$ genotype was determined by using high resolution melting (HRM) analysis. The functional cohort was used for the rs612529 genotype/phenotype association, measurement of NADPH oxidase mediated ROS production assay, ChIP assay and bisulfite treatment, and methylation analysis as described below.

\section{Genotyping}

DNA was isolated from whole blood or peripheral blood mononuclear cells (PBMCs) using DNeasy Blood \& Tissue Kits (Qiagen) according to the manufacturer's instructions. For targeted genotyping, the HRM analysis was performed by real-time polymerase chain reaction (PCR) using a CFX96 Real-Time Detection System (BioRad). The primers used for rs612529 genotyping were 5' -TCTTGGCAGAACTTCAGATAAGGT-3' (forward) and 5'-ACAAGAAGCCGTCGATGATAACT-3' (reverse). Amplification was carried out using the following protocol: 3 min at $95{ }^{\circ} \mathrm{C}, 40$ cycles of $5 \mathrm{~s}$ at $95{ }^{\circ} \mathrm{C}, 5 \mathrm{~s}$ at $50{ }^{\circ} \mathrm{C}$, and final extension for $10 \mathrm{~s}$ at $95^{\circ} \mathrm{C}$. A melting curve was generated in the range of $65-95{ }^{\circ} \mathrm{C}$ (in $0.2{ }^{\circ} \mathrm{C}$ increments) with $10 \mathrm{~s} / \mathrm{step}$. Heterozygosity and homozygosity of the allelic state were deduced using Precision Melting Analysis software (Bio-Rad).

\section{RNA extraction and global gene expression for whole blood samples}

Total RNA was extracted from human whole blood collected in Tempus Blood RNA tubes (ThermoFisher) using the MagMAX ${ }^{\mathrm{Tm}}$ for Stabilized Blood Tubes RNA Isolation Kit (ThermoFisher). Total RNA was subjected to globin mRNA depletion using the Ambion GLOBINclear kit (ThermoFisher). Agilent Bioanalyzer was used to evaluate total RNA integrity and the RNA Integrity Number (RIN) was calculated. All samples had RIN in the range of 4.7-8.8; however, the median value was 7. For genome-wide gene expression profiling, fluorescent-labeled PCR products were prepared according to Illumina Human Whole-Genome Gene Expression DASL Assay Guide. The labeled products were hybridized onto the Illumina HumanHT-12-v4 Expression Bead ChIP for $16 \mathrm{~h}$ at $58{ }^{\circ} \mathrm{C}$. These arrays were washed, coated based on the Assay Guide, and scanned using Bead Array Scanner 500GX at BSF Microarray Facility.

\section{eQTL analysis}

The whole blood cohort used for eQTL analysis has not been published yet. The median age is 21 years and 55\% male. Whole blood gene expression data were processed using the Bioconductor lumi package in $\mathrm{R}$ 3.1.2. The gene expression data were quantile normalized and log2 transformed prior to further analysis. Whole blood gene expression data for genes located 200kbp upstream and downstream of the SNP rs612529 were extracted and combined with the SNPs located within this same region. One-way ANOVAs were then done to determine if any of the SNP genotypes associated with the gene expression levels. The one-way ANOVA was conducted in $\mathrm{R}$ 3.1.2 and all resulting $P$ values were corrected for multiple testing using the method of Benjamini and Hochberg. The eQTL data for the B cells and monocytes for the 15 samples for the Chinese samples from Singapore were previously described in Melchiotti et al. [18]. Data for the B cells and monocytes for the Caucasian cohort were extracted from Fairfax et al. [19].

\section{Association analysis for rs612529}

The association analysis for the SNP rs612529 was performed using statistics from the various cohorts using Stouffer z-trend method using the Meta $P$ program (http://igm.cumc.columbia.edu/MetaP/metap.php). The Stouffer's $\mathrm{z}$ trend method considers $P$ values, sample sizes, and effect directions. For more information on the program, refer to Whitlock et al. [23].

\section{Flow cytometry analysis}

Blood samples were collected in either BD $K_{2}$ EDTA or citrate vacutainer tubes. FACS staining was performed on either using whole blood sample or isolated PBMCs. Erythrocytes were removed from whole blood by incubation in a 14-mL erythrocyte lysis buffer (155 mM $\mathrm{NH}_{4} \mathrm{Cl}, 10 \mathrm{mM} \mathrm{KHCO}, 0.1 \mathrm{mM}$ EDTA) for $10 \mathrm{~min}$ at room temperature. After erythrocytes lysis, the cells 
were centrifuged at $860 \times \mathrm{g}$ for $3 \mathrm{~min}$. The cell pellets were re-suspended in phosphate-buffered saline (PBS) and washed once with PBS by centrifugation at $860 \times$ $\mathrm{g}$ for $3 \mathrm{~min}$ at room temperature. For PBMC isolation, whole blood was layered on Ficoll-Paque (GE Healthcare) and centrifuged for $500 \times \mathrm{g}$ for $30 \mathrm{~min}$ without a break. PBMCs were harvested at the interface between Ficoll and plasma layers. To discriminate the cells live from the dead cells, lyzed whole blood samples or PBMC cell pellets were incubated with $100 \mu \mathrm{L}$ of LIVE/ DEAD Fixable Aqua Dead kit (Life Technologies) in PBS for $10 \mathrm{~min}$ at room temperature. The cells were washed once with $100 \mu \mathrm{L}$ MACS buffer (0.5\% BSA, 2 mM EDTA in PBS) and transferred into $96 \mathrm{~V}$-bottom plates for centrifugation at $1000 \times \mathrm{g}$ for $3 \mathrm{~min}$. For the SIRL- 1 phenotyping, the cell pellets were incubated with for $15 \mathrm{~min}$ at $4{ }^{\circ} \mathrm{C}$ anti-SIRL-1 FITC (1A5; as previously described [14]), anti-FceRI PE (AER37; eBioscience), anti-CD123 PerCP Cy5.5 (6H6; eBioscience), anti-CD1c BV421 (L161; Biolegend), anti-CD14 PE-CF594 (MФР9; BD Biosciences), anti-CCR3 Alexa Fluor647 (5E8; Biolegend) and anti-HLA-DR APC-H7 (L243; BD Biosciences). For the correlation of SIRL-1 with Fc receptor expression, the staining was carried out with anti-FceR1 (AER37), antiCD16 (3G8; Biolegend), and anti-CD89 (A59; BD Bioscience) monoclonal antibodies. After incubation, the cell pellets were washed once with MACS buffer, resuspended in the $250 \mu \mathrm{L}$ of the same buffer, and SIRL- 1 expression level on different cell types was determined using a LSRII or a Fortessa flow cytometer (BD Biosciences).

\section{Cell isolation}

Monocytes used for nuclear protein extraction were isolated from apheresis blood and for ChIP and bisulfite sequencing monocytes were isolated from PBMCs derived from functional cohort by positive selection using MACS Human CD14 Microbeads (Miltenyi Biotec) according to the instructions recommended by manufacturer. Neutrophils used for the bisulfite sequencing were isolated by Ficoll-Paque density gradient centrifugation. The neutrophils were isolated from the layer immediately above erythrocyte layer and lyzing the erythrocyte with erythrocyte lysis buffer $\left(155 \mathrm{mM} \mathrm{NH}_{4} \mathrm{Cl}, 10 \mathrm{mM}\right.$ $\mathrm{KHCO}_{3}, \quad 0.1 \mathrm{mM}$ EDTA) for $10 \mathrm{~min}$ at room temperature on a roller mixer. The neutrophils were washed once with cold RMPI1640 supplemented with $10 \%$ fetal bovine serum.

\section{Measurement of NADPH oxidase mediated ROS production}

ROS production was measured in primary monocytes isolated from frozen PBMC samples of genotyped donors of the functional cohort. All experiments were performed using technical duplicates. NADPH oxidase activity was, unless otherwise indicated, assessed as $\mathrm{H}_{2} \mathrm{O}_{2}$ formation and determined by Amplex Red (Molecular Probes), which reacts with $\mathrm{H}_{2} \mathrm{O}_{2}$ to produce fluorescent resorufin in the presence of HRP (Sigma-Aldrich). The assay was performed in microfluor white plates (Thermo Scientific). Isolated monocytes were re-suspended in HEPES buffer (20 mM HEPES, $132 \mathrm{mM} \mathrm{NaCl}, 6 \mathrm{mM} \mathrm{KCl}$, $1 \mathrm{mM} \mathrm{MgSO}, 1.2 \mathrm{mM} \mathrm{KH_{2 }} \mathrm{PO}_{4}, \mathrm{pH} 7.4$ supplemented

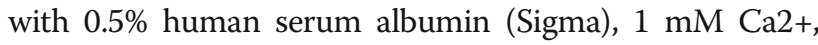
and $5 \mathrm{mM}$ D-glucose) containing $50 \mu \mathrm{M}$ Amplex Red and $1 \mathrm{U} / \mathrm{mL} \mathrm{HRP}$ and stimulated $\left(1 \times 10^{6}\right.$ cells $\left./ \mathrm{mL}\right)$ with $10 \mu \mathrm{g} / \mathrm{mL}$ plate-bound human IgA (ChromPure Human IgA, Jackson ImmunoResearch Laboratories) together with $10 \mu \mathrm{g} / \mathrm{mL}$ plate-bound anti-SIRL-1 (1A5) or an isotype-matched control mAb (functional grade mouse IgG clone:P3, eBioscience). Formation of $\mathrm{H}_{2} \mathrm{O}_{2}$ was measured directly from the start of stimulation, when cells were added to coated wells, and continued for $1 \mathrm{~h}$. Fluorescence was measured at 1-min intervals $\left(\lambda_{\mathrm{Ex}} / \lambda_{\mathrm{Em}}=545 /\right.$ $590 \mathrm{~nm}$ ) using a Tecan Infinite M200 PRO fluorescence reader (Tecan). Measurements were corrected for spontaneous $\mathrm{H}_{2} \mathrm{O}_{2}$ production, as determined by incubation of cells in non-coated wells. The percentage of SIRL-1-mediated inhibition of ROS production was calculated by sub-traction of background $\mathrm{H}_{2} \mathrm{O}_{2}$ production and subsequent calculation of the area under the curve of all samples. The AUC of IgA cocoated with an isotype-matched control mAb was set at $100 \%$ and compared with the AUC of IgA cocoated with anti-SIRL-1 mAb.

\section{Nuclear protein isolation}

Freshly isolated monocytes from leukapheresis blood were washed with ice cold PBS, then buffer A $(20 \mathrm{mM}$ HEPES, pH 7.9, 20\% Glycerol, $10 \mathrm{mM} \mathrm{NaCl}, 0.2 \mathrm{mM}$ EDTA (pH 8), 1 mM DTT, 0.1\% Triton X-100) supplemented with Pierce Proteases and Phosphatase Inhibitor Mini Tablets. The tablet contained aprotinin, bestatin, E64, EDTA, leupeptin, sodium fluoride, sodium orthovanadate, sodium pyrophosphate, beta-glycerophosphate, and metalloproteinase inhibitor. After 15 min incubation on ice, homogenates were centrifuged at $2000 \mathrm{rpm}$ at $4{ }^{\circ} \mathrm{C}$ for $15 \mathrm{~min}$, and the resultant "nuclear pellets" were resuspended in buffer A containing $500 \mathrm{mM} \mathrm{NaCl}$. The nuclear proteins were incubated for $1 \mathrm{~h}$ on ice with intermittent tapping followed by centrifugation at $13,000 \mathrm{rpm}$ $\left(4{ }^{\circ} \mathrm{C}\right)$ for $15 \mathrm{~min}$. The supernatants were then aliquoted and snap-frozen at $-80{ }^{\circ} \mathrm{C}$ until used. Quantitation of nuclear proteins was performed using Bio-Rad protein quantitation kit (Bio-Rad).

\section{Electrophoretic mobility shift assay}

The double-strand oligonucleotides had a length of 25 bp (the sequences are listed in Additional file 2). 
Allele-specific oligonucleotides corresponding to VSTM1 promoter region -34 to -58 were designed with the $\mathrm{C} / \mathrm{T}$ polymorphism of rs612529 in the center (termed as "C probe" and "T probe"). $\mathrm{C}$ and $\mathrm{T}$ probes, as well as MZF-1 consensus/mutant oligonucleotides, were synthesized by 1st BASE, Singapore; all remaining oligonucleotides were purchased from Santa Cruz Biotechnology. ${ }^{32} \mathrm{P}$ labeling was introduced using T4 Polynucleotide Kinase. For EMSA reactions, $5 \mu \mathrm{g}$ of the monocyte nuclear protein extract was incubated with $40,000 \mathrm{cpm}$ of the ${ }^{32} \mathrm{P}$-labeled oligonucleotide probe in a total volume of $20 \mu \mathrm{L} 5 \mathrm{mM}$ HEPES (pH 7.9), 5\% glycerol, $0.5 \mathrm{mM}$ EDTA, $1 \mu \mathrm{g}$ dIdC, $1 \mathrm{mM}$ DTT, and $80 \mathrm{mM} \mathrm{NaCl}$ at $4{ }^{\circ} \mathrm{C}$ for $45 \mathrm{~min}$. For "cold" competition experiments, 12.5$50 \mathrm{ng}$ of the unlabeled oligonucleotide competitor 15 min were added before adding the respective radiolabeled probe, representing a 12.5-50-fold excess of the unlabeled competitor. For supershift assays, $2 \mathrm{uL}$ of either rabbit polyclonal anti-PU.1 IgG (H-135 X, Santa Cruz Biotechnology) or rabbit polyclonal anti-YY1 IgG (C-20, Santa Cruz Biotechnology) or rabbit IgG (Abcam) were added 15 min before adding the respective radiolabeled probe. After incubation, the DNA-protein complexes were separated for $90 \mathrm{~min}$ with a $5 \%$ nondenaturing polyacrylamide gel at $240 \mathrm{~V}$ on a protein II gel-apparatus (Bio-Rad) using 0.5X Tris/Borate/EDTA (TBE). After electrophoresis, the gel was transferred to a Whatman paper and dried on a vacuum gel dryer (Gel Dryer 583, Bio-Rad) for $2 \mathrm{~h}$ at $80{ }^{\circ} \mathrm{C}$. The dried gel was then exposed on an imaging plate and scanned using a FLA-5000 phospho-image scanner (Fujifilm Life Science).

\section{ChIP assay}

ChIP assays were performed on monocytes isolated from four CC-donors and five TT-donors (rs612529) of the functional cohort. ChIP was carried out essentially as previously described [24]. Briefly, DNA/protein crosslinking was achieved by incubating the cells for $8 \mathrm{~min}$ at $37{ }^{\circ} \mathrm{C}$ in $1 \%$ formaldehyde. Ultrasound sonication of the chromatin was performed in lysis buffer $(50 \mathrm{mM}$ Tris-Cl $\mathrm{pH}$ 8.0, $10 \mathrm{mM}$ EDTA, 1\% SDS) with 3-min cycles (30 s "ON", $30 \mathrm{~s}$ "OFF") for a total of ten cycles using a Bioruptor UCD-300 sonicator (Diagenode). After sonication, samples were diluted in fivefold dilution buffer $(0.01 \%$ SDS,1.1\% Triton X-100, $1.1 \mathrm{mM}$ EDTA, $20 \mathrm{mM}$ Tris-Cl $\mathrm{pH}$ 8.0) followed by overnight incubation at $4{ }^{\circ} \mathrm{C}$ with $10 \mu \mathrm{g}$ of either rabbit polyclonal anti-PU.1 antibody IgG (H-135 X, Santa Cruz Biotechnology), or rabbit polyclonal IgG (Abcam). Real-time PCRs of genomic regions containing the putative PU.1-binding site were performed in triplicate by using iTaq SYBR green supermix (Bio-Rad) with SIRL-1 specific primers (forward primer: 5' -TCTTGGCAGAACTTCAGATAAGGT-3'; reverse primer: 5' -ACAAGAAGCCGTCGATGATAACT-3'). The relative occupancy of the immunoprecipitated factor at SIRL-1 locus is estimated by using the comparative threshold method $2^{\wedge}\left(\mathrm{Ct}_{\text {mock }}-\mathrm{Ct}_{\text {specific }}\right)$, where $\mathrm{Ct}_{\text {mock }}$ and $\mathrm{Ct}_{\text {specific }}$ are mean threshold cycles of PCR done in triplicate on DNA samples from mock and specific immunoprecipitation [24].

\section{Bisulfite treatment and DNA methylation analysis}

Primers for the bisulfite genomic sequencing (BGS) were designed to cover a region 361 bp upstream and 335 bp downstream to the transcription start site of VSTM1 (Additional file 3). DNA bisulfite treatment of the genomic DNA isolated from freshly isolated monocytes and neutrophils of respectively four donors of the CC and TT genotype (functional cohort) was performed according to the protocol described in EZ DNA MethylationDirect $^{\mathrm{Tm}}$ Kit (Zymo Research). For BGS, bisulfite-treated DNA was amplified using the SIRL-1 promoter specific primers

(F1: 5' -AGGTTGGAGTGTAGTGGTATAATTTTGG-3'; F2: 5' - ACTCTAACACTCTAATTCTCTACCCCAC-3',; B1:5' AGAGTGGGGTAGAGAATTAGAGTGTTA and

B2: 5' - ACCTAAACTACCACTCCCACATAAAT-3') with optimized conditions. The PCR products were cloned into the TA cloning vector (Invitrogen, Life technologies). For both monocytes and neutrophils three clones from each donor and sequenced using M13 forward primer.

\section{CpG islands (CGI) prediction}

A Hidden Markov Model (HMM) based CGI prediction for the VSTM1 gene locus was obtained from UCSC genome browser as custom tracks (http://genome.ucsc.edu/ cgi-bin/hgTracks) [25]. CpG islands (CGI) from DNA sequences and custom tracks were uploaded from Rafael Irizarry lab (http://rafalab.jhsph.edu/CGI/).

\section{Results}

VSTM1 promoter-associated SNP rs612529 is an eQTL for SIRL-1

We used whole blood eQTL data of 202 ethnic Chinese donors (unpublished dataset) collected in Singapore to determine if our previously reported inter-individual variations in SIRL-1 levels $[12,16]$ are linked to SNPs. The results described in Fig. 1a reveal a strong eQTL peak at the promoter of the VSTM1 gene that encodes for SIRL1 (all cohorts used in the study are described in Additional file 1). The effect was caused by a single SNP (rs612529T/C) located $45 \mathrm{bp}$ upstream of the transcriptional start site (TSS) in the promoter region of VSTM1 $\left(P=8.13 \times 10^{-13}\right)$. We also used our previous data [18] based on gene array analysis of monocytes and B cells isolated from a genotype matched Chinese cohort of 15 individuals. Primary isolated monocytes from the $\mathrm{T}$ allele 
a

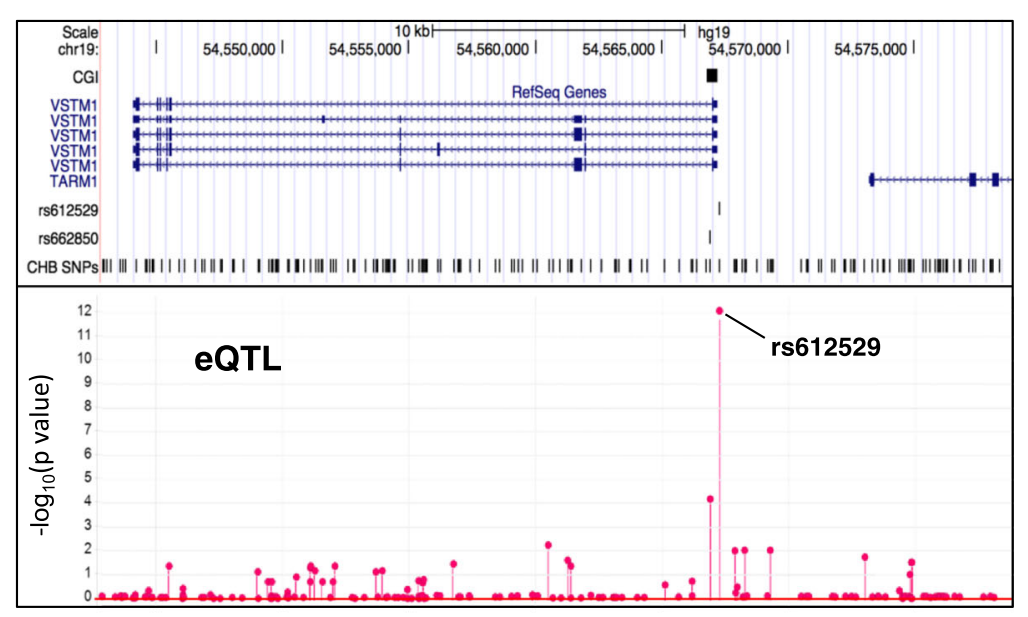

b

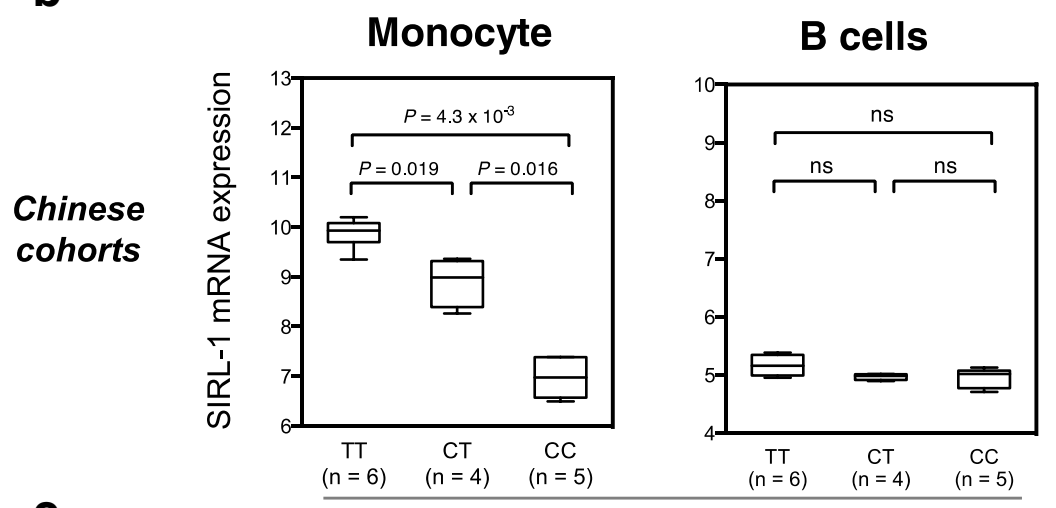

C

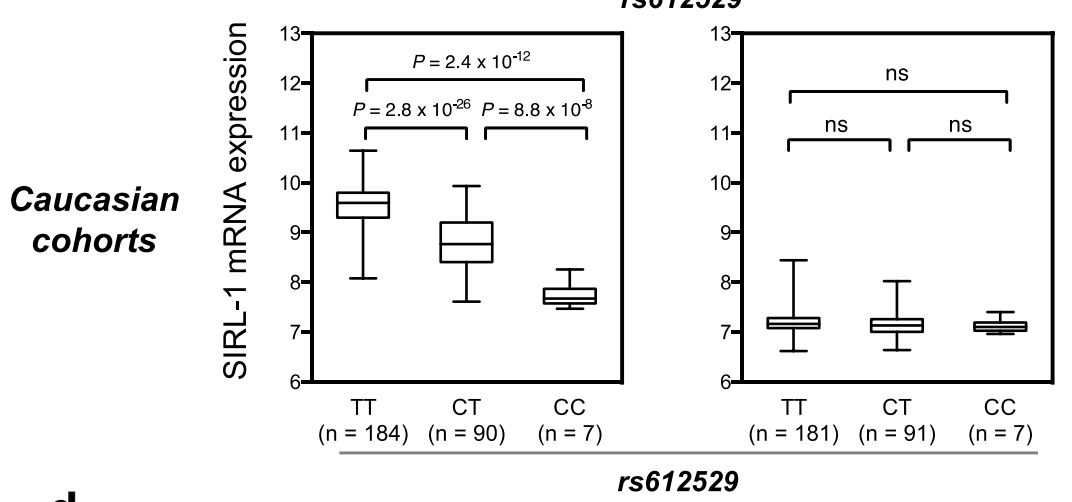

d
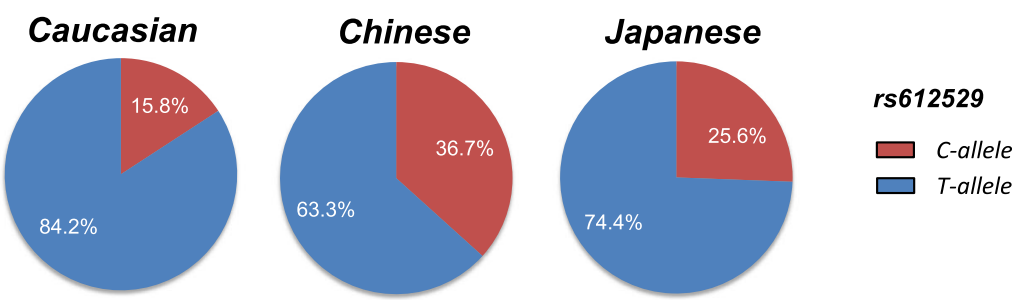

Fig. 1 (See legend on next page.) 
(See figure on previous page.)

Fig. 1 rs612529 is an eQTL affecting SIRL-1 expression. a Manhattan plot of whole blood eQTLs located in the SIRL-1-encoding VSTM1 gene region. The upper panel depicts the position of VSTM1 transcripts in relation to a CGI and the SNPs rs612529 and rs662850. The lower panel shows the eQTL-association determined from whole blood samples of 202 Chinese individuals. The $P$ value (expressed as -log 10 ) is displayed on the $y$-axis, the $x$-axis shows the location of the SNPs (rs612529 is indicated). b, c Genotype effect of rs612529 on the SIRL-1 mRNA expression monocytes and B cells. The data were generated from cohort samples of 15 Chinese [18] and 281 Caucasian individuals [19]. The number of individuals per genotype is indicated on the $x$-axis; the $y$-axis represents the SIRL-1 mRNA expression levels depicted as log 2 value. $\mathbf{d}$ Allele frequencies of rs612529 in three major ethnical groups. The pie charts display the frequencies of the C allele (red) and the T allele (blue) for Caucasian, Han Chinese, and Japanese based on HapMap

donors exhibited a higher level of SIRL-1 mRNA expression, while the level was considerably lower in the $\mathrm{C}$ allele donors (Fig. 1b). The mRNA levels gradually increased from the $\mathrm{CC}$ over $\mathrm{CT}$ to TT genotype, resulting in a more than tenfold difference between the two homozygous genotypes of $\mathrm{CC}$ versus TT $\left(P=4.30 \times 10^{-3}\right)$. We validated this finding in a much larger dataset of 283 Caucasian individuals based on published eQTLs [19] $\left(P=2.40 \times 10^{-12}\right)($ Fig. 1c). The low expression allele was less frequent in the Caucasians with allele frequency of the $\mathrm{C}$ allele in Caucasian at $15.8 \%$ compared with $36.7 \%$ in Chinese and 25.6\% Japanese (Fig. 1d). No significant eQTL association was observed for VSTM1 mRNA in B cells isolated from the same donors in both datasets (Fig. $1 \mathrm{~b}$ and $\mathrm{c}$ ).

\section{Cell-type-specific regulation of SIRL-1 protein expression in monocytes}

Our eQTL association for rs612529 seems to affect VSTM1 expression monocytes and not B cells. SIRL-1 is expressed on various immune cells of the myeloid lineage. To determine the effect of rs612529 monocyte SIRL-1 at the protein level, we used flow cytometry to analyze three genotype donors (Fig. 2a). Scatter analysis with anti-SIRL-1 staining revealed allele-dependent effects on the amount of SIRL-1 surface expression only on monocytes. Notably, no influence of the SNP was observed for the granulocyte subset (Fig. 2a). On these cells a high expression of the receptor was detected independent of the genotype of rs612529. We then validated the finding in a cohort of 44 donors by using a staining panel of seven antibodies (Fig. 2b). The gating strategy (Additional file 4) allowed the simultaneous quantification of the SIRL-1 expression on various cells of the myeloid lineage (monocytes, neutrophils, eosinophils, basophils, myeloid dendritic cells (mDC), and plasmacytoid DC (pDC) as well as on lymphocytes (B cells, natural killer (NK) cells/T cells) (Fig. 2c). The analysis confirmed the strong genetic association with SIRL-1 surface expression on monocytes $\left(P=2.4 \times 10^{-9}\right)$ as well as the high but genotype-independent expression on neutrophils. A slight effect of the rs612529 polymorphism was observed also on eosinophils and basophils ( $P=0.0358$ and $P=0.0077$, respectively), while on
mDC, pDC, B cells, and NK cells/T cells, the SIRL-1 expression fell below detection limit (Fig. 2c).

\section{Impact of rs612529 on the inhibition of ROS}

We have shown previously that SIRL-1 inhibits the FcRmediated respiratory burst $[12,16]$. In order to determine the functional implications of the polymorphism, we analyzed the antibody-induced ROS production in monocytes based on the genotype of rs612529. Depending on differentiation and maturation, monocytes express varying amounts of FcR specific for IgG (FcyRIII, CD16), IgE (FceRI), and IgA (Fc $\alpha R, C D 89)$. Our flow cytometry analysis on ex vivo monocytes revealed that the SIRL-1 expression was highest on monocytes expressing the Fc receptor for IgA (Fig. 3a, Additional file 5). More than $90 \%$ of the Fc $\alpha R+$ cells co-expressed SIRL-1 (Fig. 3a, right panel). Hence, for the follow-up ROS analysis, the FcR-induced respiratory burst was induced using immobilized IgA. The resulting data, as shown in Fig. 3b, demonstrate that exposure of primary monocytes to IgA resulted in a strong production of ROS. Simultaneous ligation of SIRL-1 with an agonistic antibody resulted in an rs612529 allele-dependent reduction of the levels of detectable ROS. This was confirmed with monocytes isolated from a cohort of 30 genotyped individuals (Fig. 3c). A gradual decrease in the inhibition from TT over TC to $\mathrm{CC}$ was observed, a trend that matched the decreasing amount of SIRL-1 on the surface of these cells. This was particularly evident in a direct correlation between the amount of SIRL-1 on the surface of the cells and the inhibition of the IgA-induced ROS production (Fig. 3d).

\section{rs612529 regulates the recruitment of PU.1 and YY1 to the VSTM1 promoter}

In order to determine if rs612529 modulates the binding of essential transcription factor(s) we carried out EMSAs. EMSA experiments performed with monocytederived nuclear samples revealed allele-specific binding of at least two different factors (Fig. 4a). Both factors bound selectively to the $\mathrm{T}$ allele ("T probe") but not the $\mathrm{C}$ allele ("C probe") of an oligonucleotide probe representing the $21 \mathrm{bp}$ promoter segments of the SNP. Allele specificity of the binding was confirmed by cold 
a

Full blood analysis

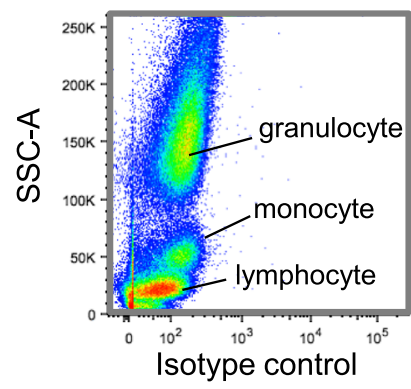

b
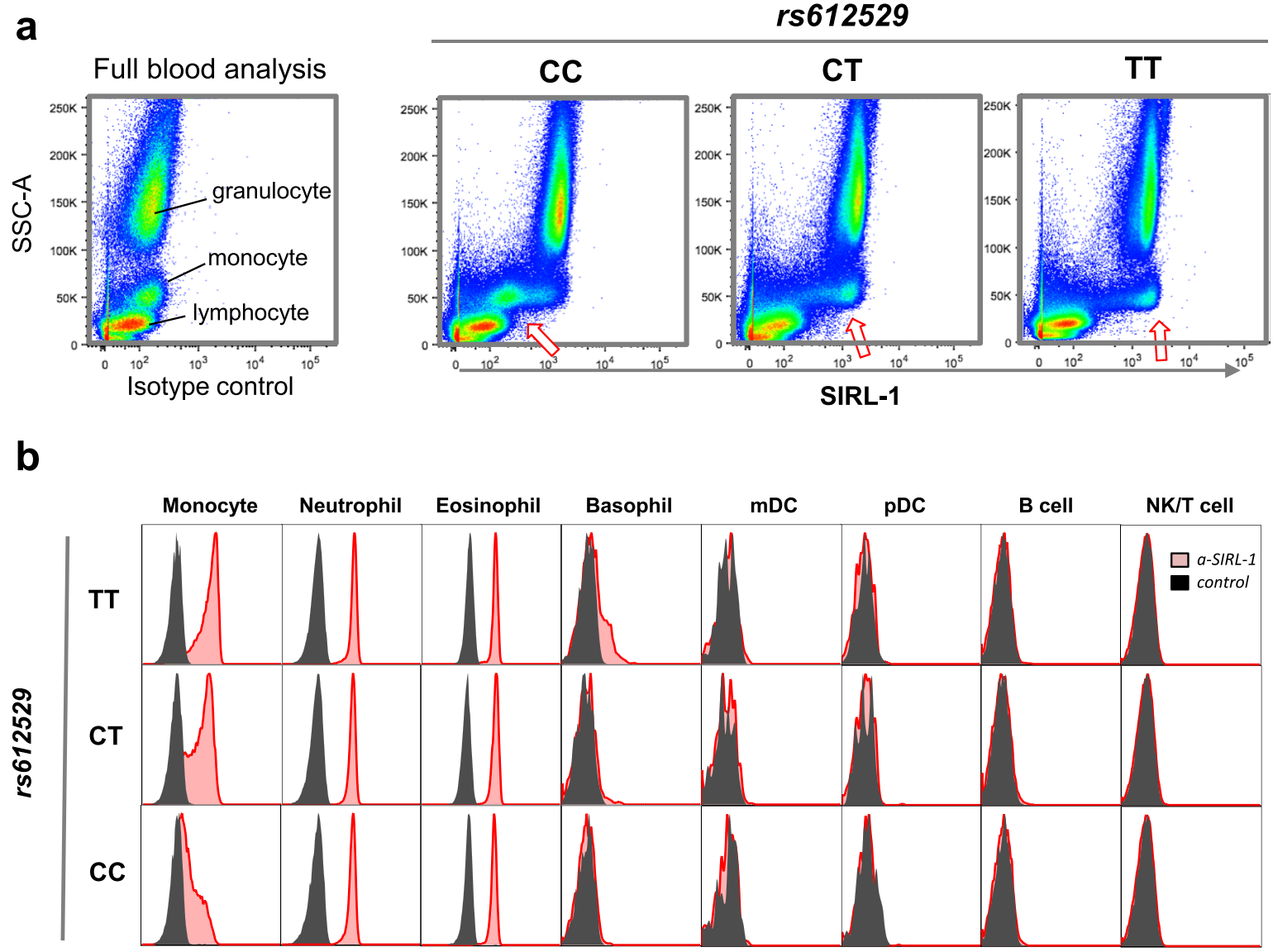

C

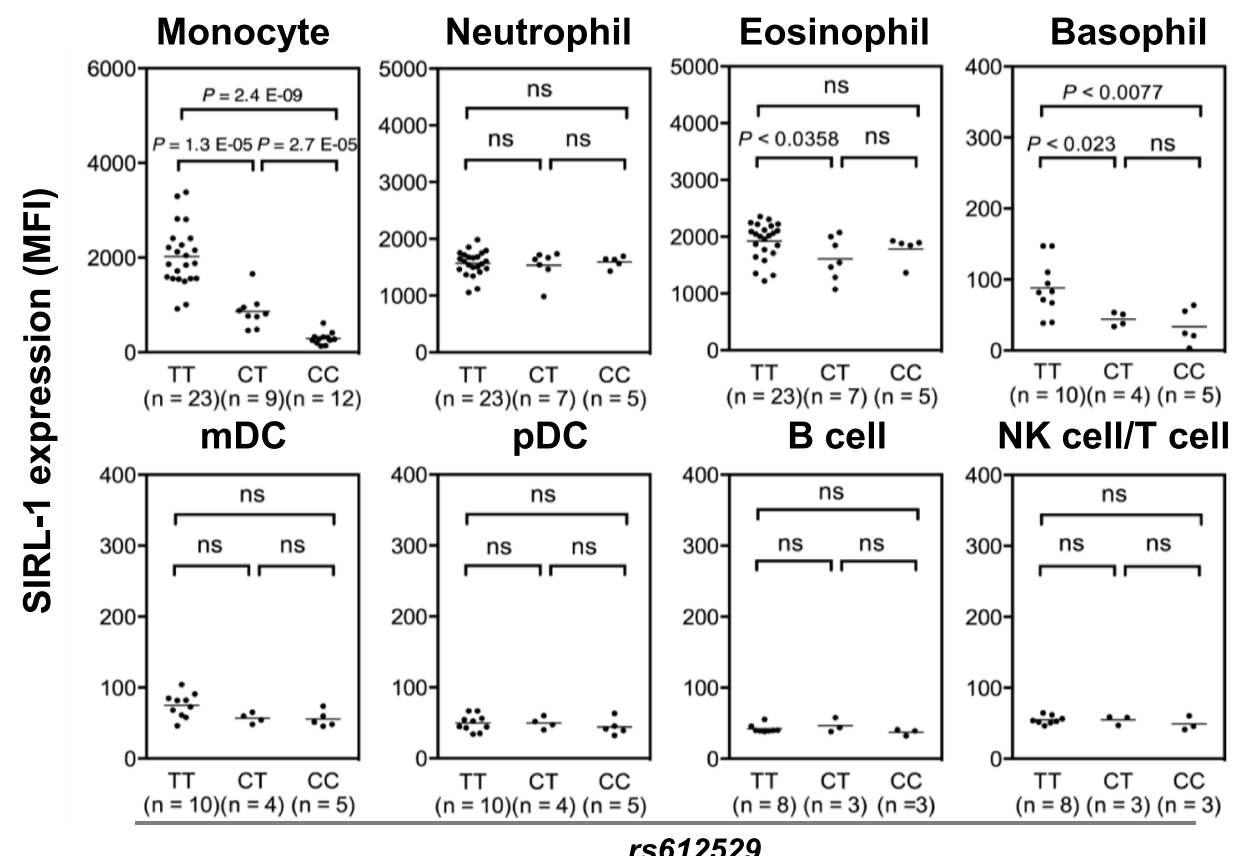

Fig. 2 (See legend on next page.) 
Kumar et al. Genome Medicine (2017) 9:18

Page 9 of 16

(See figure on previous page.)

Fig. 2 Cell-type-specific effect of rs612529 on SIRL-1 surface expression. a Flow cytometry analysis of whole blood samples. Whole blood samples were stained with a SIRL-1 specific antibody (right panel) and an isotype-matched control antibody (left panel). The staining is shown vs. the side scatter (SSC-A), which allows a simple discrimination of granulocytes, monocytes, and lymphocytes. The plots are representative examples of individuals with the rs612529 genotype TT, TC, and CC. Arrows indicate the gradual increase in SIRL-1 staining on monocytes. b Genotypedependent SIRL-1 expression on various cell types. SIRL-1 staining (red) in reference to the isotype control (black) is shown for each of the three rs612529-genotypes for myeloid cells (monocyte, neutrophil, eosinophil, basophil, aDC, and DC) and two lymphocyte subsets (B cell, NK cell/T cell). Data were generated by flow cytometry from whole blood samples after gating on the respective cell subset (gating strategy is displayed in Additional file 4). c Cohort-wide distribution of the SIRL-1 expression. The dot plots summarize the SIRL-1 FACS data for a cohort of 44 genotypematched individuals

a

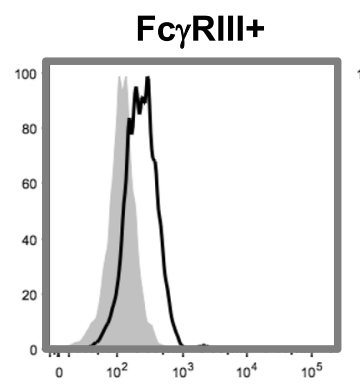

FeRIa+

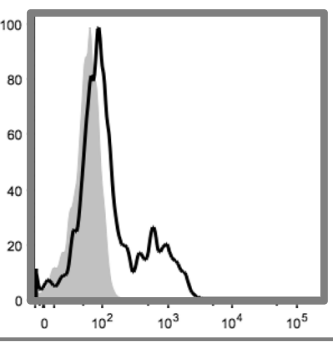

SIRL-1
FeaR+

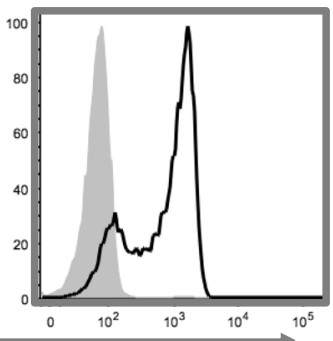

b

rs 612529
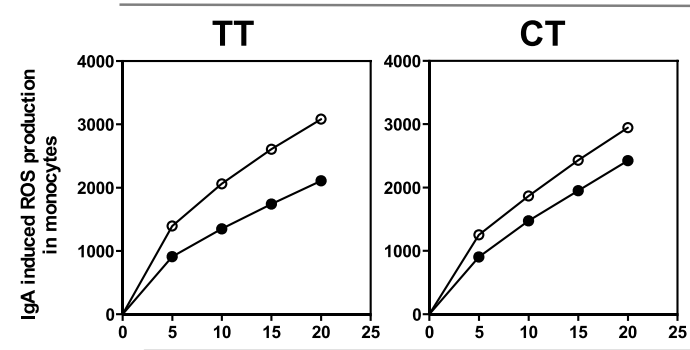

CC

Time

C

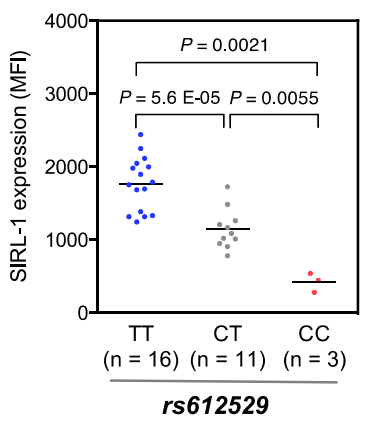

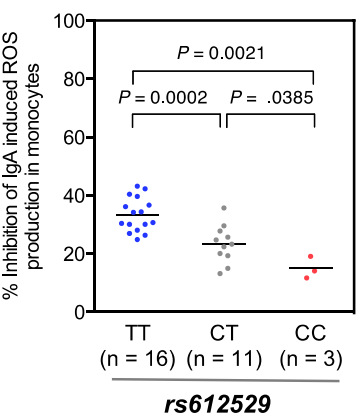

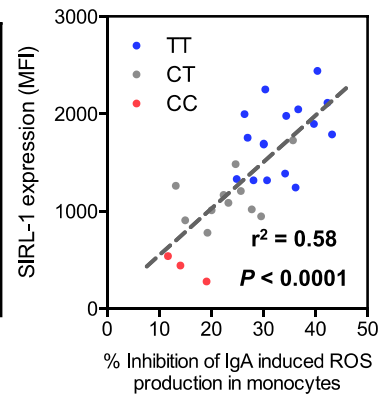

Fig. 3 Allele-specific inhibition of IgA-induced ROS production by SIRL-1. a Comparison of the SIRL-1 expression on monocytes expressing FcyRIII-, FcERla- or FcaR-receptors. Flow cytometry analysis of the SIRL-1 surface expression is shown for monocytes gated on the expressing of the Fc-receptors FcyRIII, FcERI or Fca (gating strategy is shown in Additional file 5). The SIRL-1 specific staining (black lines) is shown in reference to the isotype control ( ray). b Allele-dependent inhibition of ROS production. FcaR-mediated ROS production is shown for primary monocytes induced by plate-bound IVA in the presence of agonistic anti-SIRL-1 antibodies (filled circle) or isotype-matched control antibody (open circle). Representative examples are shown for each of the three rs612529-genotypes. ROS production was measured by ELISA at $\lambda_{E x} / \lambda_{E m}=545 / 590 \mathrm{~nm}$ using $\mathrm{H}_{2} \mathrm{O}_{2}$ sensitive Amplex Red; the units represent background corrected relative fluorescence unit (RFU). c ROS inhibition obtained for a cohort of 30 genotype donors. The plots display the SIRL-1 surface expression (left panel) and the percent inhibition of the IgA-induced ROS production (right panel) in reference to the respective rs 612529 genotype. $\mathbf{d}$ The correlation of SIRL-1 expression with the inhibition of IgA-induced ROS production. The correlation shown is 30 individuals. Dashed line represents the linear regression. rs612529 genotype is indicated by the color code (TT: blue, CT: gray; CC: red) 

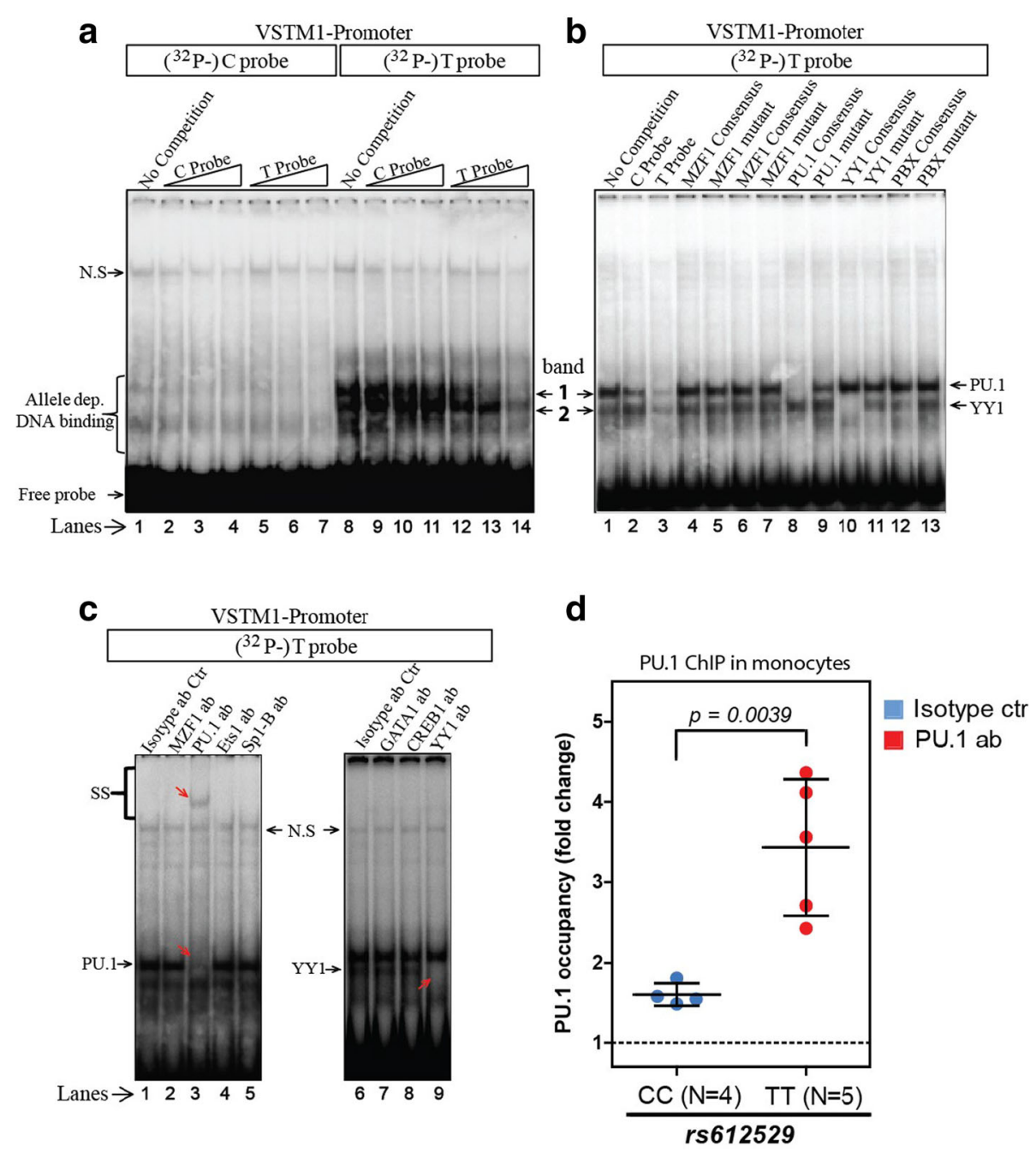

Fig. 4 rs612529 C/T controls the binding of PU.1/YY1 to the VSTM-1 promoter. a Allele-specific binding to rs612529. EMSA experiments with nuclear extracts from monocytes were carried out with radiolabeled probes representing the $C$ allele ("C probe;" lanes $1-7)$ or the $T$ allele of rs612529 ("T probe;" lanes 8-14). In a "cold" competition, the binding to the radiolabeled probes was competed with unlabeled C probes (lanes 2-4 and 9-11) or T probes (lanes 5-7 and 12-14). Bands 1 and 2 are two bands formed by allele-specific binding; "N.S." indicates a non-specific band. $\mathbf{b}$ Competition with consensus binding motifs of predicted candidate factors. Binding to the T probe was competed with unlabeled probes representing consensus binding motifs for MZF-1, PU.1 and YY1 (PBX was used as control). Binding of each factor was competed with the optimal consensus sequence as well as a mutated variant to which the binding was abolished (sequences are listed in Additional file 7). c Super-shift assays. Super-shift assays were carried out with the T probe and antibodies against MZF-1 (lane 2), PU.1 (lane 3), Ets1 (lane 4), Sp1-B (lane 5), GATA1 (lane 7) CREB1 (lane 8), and YY1 (lane 9) or isotype-matched control antibodies (lanes 1 and 6). Super-shifted bands and bands lost by the antibody treatment are indicated by red arrows. d PU.1-ChIP assay. A ChIP assay with nuclear samples from monocytes of four CC and five TT donors (rs612529) was carried out with PU.1-specific antibodies (red dots) or isotype-matched control antibodies (blue dots). PU.1 occupancy, depicted as fold change compared to the isotype, was determined by quantitative PCR amplification of the respective region in the VSTM-1 promoter (Additional file 3)

competition experiments, since unlabeled $\mathrm{T}$ probe could out-compete both factors, while the unlabeled $\mathrm{C}$ probe had no effect on their binding to the ${ }^{32}$ P-labeled $\mathrm{T}$ probe.

In silico analysis by JASPAR (http://jaspar.binf.ku.dk/) and TRANSFAC (http://www.gene-regulation.com/) predicted binding by PU.1, MZF-1, and YY1 (Additional file 6). EMSA competition of the radiolabeled $\mathrm{T}$ probe with unlabeled oligonucleotides representing the consensus binding sequences of these transcription factors suggested band 1 to be formed by PU.1, while band 2 derived from YY1 (Fig. 4b; Additional files 2 and 7). The identity of the two factors was formally confirmed by super-shift assays, in which the PU.1-specific and YY1specific antibodies super-shifted and/or blocked the complex formation with the $\mathrm{T}$ probe (Fig. 4c).

The allele-specific interaction of PU.1 with the VSTM1 promoter region in monocyte-derived nuclear samples 
was also confirmed in ChIP assays (Fig. 4d). DNA fragments precipitated from four CC and five TT donors with PU.1-specific antibodies were analyzed by quantitative PCR with a primer pair covering a region of the VSTM1 promoter including the rs612529 SNP (Additional file 3). In line with the results from the EMSA experiments, a significant increase in the amount of VSTM1 DNA was detected in the PU.1 precipitate from TT donors compared to CC donors (Fig. 4d). Thus, in monocytes PU.1 is strongly associated with the analyzed VSTM1 promoter segment when rs612529 consists of the $\mathrm{T}$ allele.

\section{The allelic state of rs612529 is correlated with the methylation of VSTM1 in monocytes}

Epigenetic mechanisms facilitate the response to the changes in the environment through modulating the gene expression. For example, the methylation of $\mathrm{CpG}$ pairs triggers condensation of the DNA, which affects the interaction with transcription factors and other regulatory factors controlling gene expression [26]. This mechanism seems to be relevant also for VSTM1 as in lymphoma cells (which do not exhibit any SIRL-1 expression) the silencing of the gene is associated with the methylation of some CpG pairs located within the first exon [27]. An in silico scan (http://rafalab.jhsph.edu/ CGI/) [25] suggested the presence of a CGI at the start of VSTM1. It completely covered exon 1, extending from the promoter region towards intron 1 (Fig. 5a). A segment of that region of about $700 \mathrm{bp}$ (411 bp upstream and $282 \mathrm{bp}$ downstream of the TSS contained a total of 30 CpG pairs including one CpG-SNP formed by the C allele of rs612529 (Additional file 3).

In order to study a potential role of the rs612529 CpG-SNP in the control of the monocyte-specific SIRL1 expression, we analyzed cytosine methylation by bisulfite sequencing. A total of eight donors (four CC genotype and four TT genotype) were used to determine the influence of the allelic state in both eQTL relevant monocytes and allele-independent neutrophils (Fig. 5b and c). While the CpG-SNP itself would appear to be a potential site for an allele-specific methylation, it was apparently not a target for modification, as it was found to be located in a 54-bp core region containing six additional CpG pairs, which were constitutively demethylated in all samples analyzed. While the CpG-SNP was not directly affected by epigenetic modifications, it had a strong and cell-type-specific effect on the general methylation state in the analyzed gene region. In line with the bright but allele-independent surface expression detected by FACS (compare Fig. 2) neutrophils exhibit demethylated CpG elements both in the CC and the TT donors (Fig. 5b). In monocytes, however, only TT donors had a demethylated VSTM1 promoter, while the gene segment was almost completely methylated in $\mathrm{CC}$ donors (Fig. 5c). Thus, in monocytes the formation of a CpG pair by the $C$ allele of rs612529 seems to promote the methylation of other CpG pairs in the region results in a silencing of the locus (compare Fig. 2).

\section{Genetic association of rs612529 to AD phenotype}

From earlier reports, it was hypothesized that allergic and inflammatory skin diseases are linked to oxidative stress [28, 29]. Since SIRL-1 acts as a balancing factor for the antibody-induced ROS production by monocytes, we evaluated whether the allelic state of rs612529 had any influence on the manifestation of inflammatory skin diseases. We used an existing allergy cohort for rs612529 genotype association studies $[20,30]$ (Additional file 1). Association analysis for rs612529 in 324 clinically diagnosed AD cases and 486 healthy controls identified a significant association of $P<0.05$ with the $\mathrm{C}$ allele present at a higher frequency in patients than in healthy controls $\left(\mathrm{OR}=1.3 ; P=4.00 \times 10^{-6}\right)$. The same trend was also observed in two larger cohorts, one Chinese [21] and one Caucasian [22] (Table 1). Upon combining all three independent cohorts, the association of rs612529 to AD risk remained significant with a Meta $P$ value of $1.14 \times 10^{-6}$ in a combined sample size of 2536 AD cases against 4118 controls. Thus, the low surface expression of SIRL-1 in monocytes associated with the $\mathrm{C}$ allele potentially increases risk for inflammatory skin diseases such as dermatitis.

\section{Discussion}

In this study, we significantly associate the previously reported cell-type-specific variations in SIRL-1 expression to a genetic cause. A single SNP located in the promoter region mediates the epigenetic silencing of the entire gene locus, a process presumably mediated by the allelespecific recruitment of PU.1/YY1. The allelic effect is particularly evident in monocytes, where the $\mathrm{C}$ allele of rs612529 is associated with a near complete lack of SIRL-1 expression. This in turn results in a diminished control of pro-inflammatory reactions, evidenced by the failure of SIRL-1 agonists to dampen the production of Fc-receptor induced ROS in these cells. Genetic association results in independent cohorts suggest that this is associated consistently with an increased risk of AD.

We then demonstrated allele-specific binding of PU.1 to the promoter region of VSTM1 using follow-up functional experiments such EMSA and ChIP assays. This transcription factor PU.1 has been shown to be a master regulator of myeloid cell development, which could explain the observed specificity of the SIRL-1 expression in cells of this lineage [31-33]. PU.1 forms complexes with various regulatory factors chromatin remodelers, including DNA methyltransferases and demethylases to 
a

b

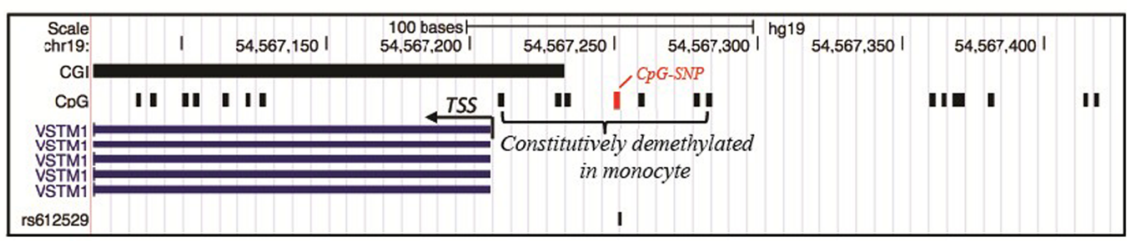

Neutrophils (CC genotype)

rs612529 (C)

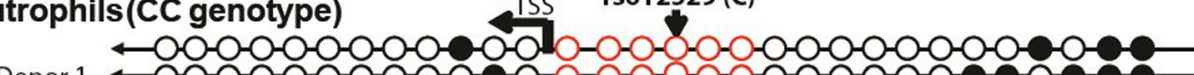

Donor 1 ־0-0-0-0-0-0-0-0-0-0-0-0-0-0-0-0-

- - - - - - - - - - - - - - - - - - - - - - - -

Donor 2 घ0-0-0-0-0-0-0-0-0-0-0-0-0-0-0-0-0-

Donor 3 ㅇ-o-0-0-0-0-0-0-0-0-0-0-0-0-0-0-0—م-0-0-0-0-0-0-0-0-0-0-0-0-0-0-0-0-0-0-0-

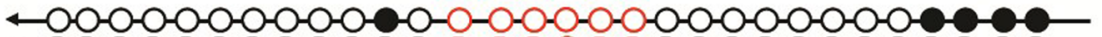

Donor 4 «0-0-0-0-0-0-0-0-0-0-0-0-0-0-0-0-0-

Neutrophils(TT genotype) rs612529 (T)

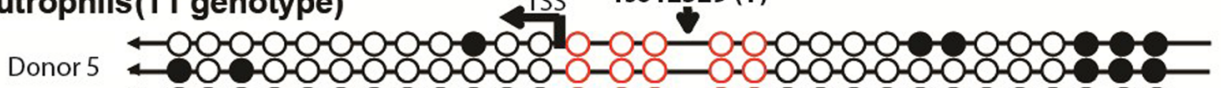
=0-0-0-0-0-0-0-0-0-0-0-0-0-0-0-0-0-0-0-

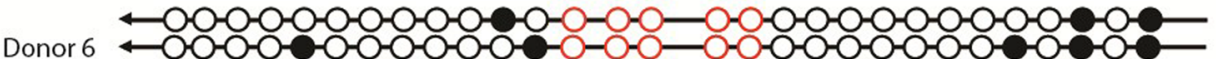
—0-0-0-0-0-0-0-0-0-0-0-0-0-0-0-0-0-0-0-0-0$\longleftarrow$ -

Donor 7 -00-0-0-0-0-0-0-0-0-0-0-0-0-0-0-0-0-0-0-00-0-0-0-0-0-0-0-0-0-0-0-0-0-0-0-0-0-0-0-0-0-0-

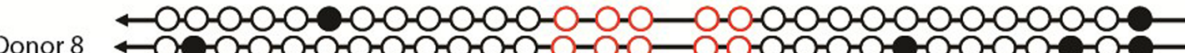

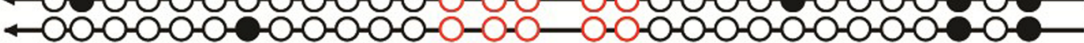

C

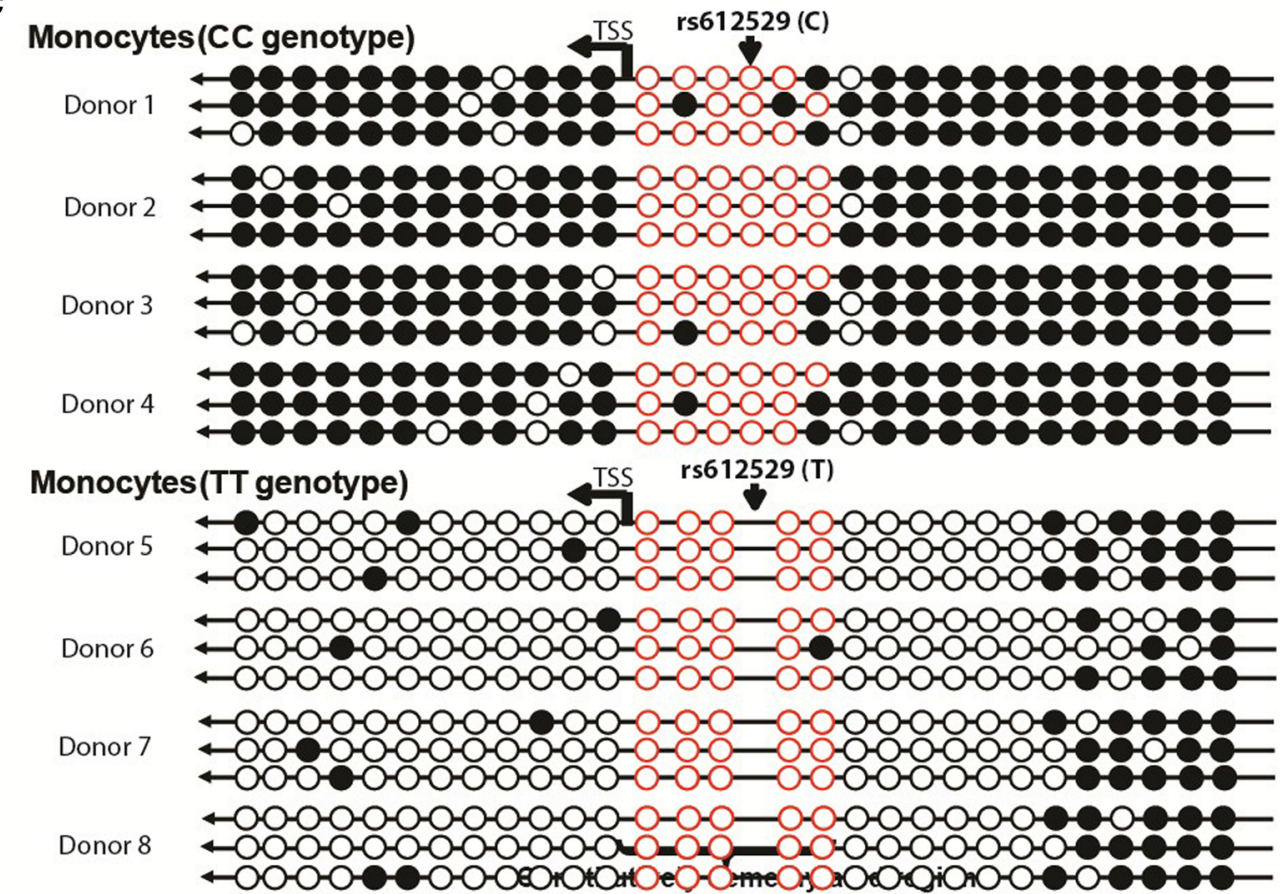

Fig. 5 (See legend on next page.) 
(See figure on previous page.)

Fig. 5 Allele and cell type-dependent methylation of the VSTM1 gene promoter. a Schematic overview of the analyzed 693 bp region of the VSTM1 gene locus ( -1 to -388 upstream and +1 to +305 downstream to TSS). The location of a predicted CGI is indicated together with the position of individual CpG pairs (solid black lines); the CpG-SNP formed by rs612526 is indicated by a red box. The location of the first exon in the transcript variants of VSTM1 (covered in the C-methylation assay) is represented by solid blue vertical lines (the sequence of the 693 bp region is shown in Additional file 3). b Allele-independent C-methylation in neutrophils. The methylation of CpG pairs in the 693 bp region was analyzed by bisulfite sequencing. The methylation state of each CpG element is represented by circles (full circle: methylated, empty circle: demethylated); the location of the TSS as well as of rs612529 is indicated. The methylation state in the cells is shown as a set of three independently sequenced clones each obtained from four different donors of the rs612529 CC genotype (donors 1-4) and the TT genotype (donors 5-8). Red circles indicate CpG pairs of the constitutively demethylated core region. c Allele-dependent methylation in monocytes. The CpG-methylation state is shown for monocytes, which were isolated from the same donors described above for the neutrophils

activate or repress transcription [34]. A recent study has shown that in non-differentiated monocytes, PU.1 can act as docking site for the demethylase, Tet2 [35]. In these cells the recruitment of Tet2 by PU.1 leads to the targeted demethylation of the respective gene locus. In line with this observation we therefore hypothesize that the allele-dependent methylation of the VSTM1 gene reported here can be explained by the conditional recruitment of Tet2 or other demethylases as a result of the allele-specific binding of PU.1 to rs612529 (Additional file 8). In monocytes rs612529 would thus represent a genetic master switch for the epigenetic control of SIRL1 expression by $\mathrm{CpG}$ demethylation.

Mechanistically, the methylation of $\mathrm{CpG}$ promotes the compaction of chromatin by providing interaction sites for methyl-CpG-binding proteins (MBPs) [24, 36, 37]. Hence, CpG-SNPs, which allow methylation in an alleledependent way, are often associated with function [38-40]. In this context, it is interesting to note that rs612529 is a CpG-SNP where the $\mathrm{C}$ allele forming the $\mathrm{CpG}$ pair is associated with the epigenetic silencing of the VSTM1 gene. However, in contrast to conventional CpG-SNPs, it is not a direct target for methylation. Instead of acting as a simple "switch" to close the chromatin, it rather functions as a genetic/epigenetic regulator that modulates the DNA methylation state by the recruitment of CpG modifying enzymes. In this way, the effect of the CpG-SNP becomes potentiated, as the methylation affects not just a single CpG but also many other $\mathrm{CpG}$ sites in its vicinity. Subtle changes associated with the allelic switching between $\mathrm{C}$ and $\mathrm{T}$ therefore translate into extensive epigenetic alterations of the gene, which makes rs612529 a master genetic regulator of the VSTM1 gene in monocytes.

We then evaluated if the causal SNP we identified, rs612529, has potential pathological implications, given the striking variations in SIRL-1 surface expression observed on monocytes. By using the allelic states of the SNP as proxy for the expression levels in these cells we could establish AD as being associated with a low SIRL1 expression in these cells. AD is a chronic inflammatory skin disease affecting 60 million people in Europe and the USA [41]. The pathophysiology of $\mathrm{AD}$ is a result from a complex interplay of host genetic factors, environmental triggers, skin integrity, immune balance, and the skin microbiome [42]. Thus, a tight control of the immune response is needed to ensure effective pathogen defense without collateral damage to the protective skin layers. IgE is one of the determinants predisposing factor for AD. However, earlier reports have shown that in early childhood, elevated levels of IgE do not seem to correlate with disease manifestations or severity of $\mathrm{AD}$ [43]. Moreover, some evidence pointing towards a possible role of IgA derives from an earlier report indicating that patients with selective IgA deficiency have an increased incidence of atopic dermatitis [44].

Table 1 Association of rs612529 with AD

\begin{tabular}{|c|c|c|c|c|c|c|c|}
\hline References $^{a}$ & Population $^{\mathrm{b}}$ & Cases $^{c}(n)$ & Controls $^{\mathrm{d}}(\mathrm{n})$ & Effect allele ${ }^{e}$ & Effect allele frequency ${ }^{f}$ & OR $(95 \% \mathrm{Cl})^{9}$ & $P$ value $^{h}$ \\
\hline [15] & Caucasian & 1200 & 2270 & C & 0.209 & $1.12(1.15-1.29)$ & $1.26 \times 10^{-3}$ \\
\hline [14] & Chinese & 1012 & 1362 & C & 0.322 & $1.15(1.07-1.30)$ & $2.6 \times 10^{-2}$ \\
\hline \multirow[t]{2}{*}[12,13]{} & Chinese & 324 & 486 & C & 0.36 & $1.29(1.10-1.45)$ & $4 \times 10^{-6}$ \\
\hline & Combined & 2536 & 4118 & & & & $1.14 \times 10^{-6}$ \\
\hline
\end{tabular}

anitial study describing the cohorts used for association

${ }^{b}$ Ethnicity of the population under study

cNumber of atopic dermatitis cases

${ }^{\mathrm{d}}$ Number of controls

eAllele used for the calculating the odds ratio statistics

${ }^{f}$ Allele frequency of the effect allele in the individual study population

${ }^{g}$ Odds ratio estimated with $95 \%$ confidence interval

${ }^{\mathrm{h}} P$ value estimated for the case control association study for atopic dermatitis phenotype in the individual study population

Meta $P$ value is the $P$ value calculated for all three cohorts combined using the Stoufier's $z$ trend meta-analysis which considers individual study $P$ value sample sizes and effect directions using Meta $P$ program [20] 
SIRL-1 is an effective inhibitor of monocyte-derived pro-inflammatory reactions such as FcR-induced ROS production. In our study, we show that blood monocytes represent a heterogeneous population characterized by different Fc receptors. In Fig. 3a and Additional file 5, FcaR + monocytes co-segregate with a high expression of SIRl-1 expression. This is not observed in FcyRIII+ and Fce $R+$ monocytes, the latter being expressed on a very small subset of monocytes. As such, we examined if coactivating of FcaR and SIRL-1 with human IgA and antiSIRL-1 mAb would influence the capacity of monocytes to generate ROS. We show the capacity of SIRL-1 to modulate ROS production in monocytes is a function of VSTM1 genotype. The association of the low expressing $\mathrm{C}$ allele with $\mathrm{AD}$ thus leads us to hypothesize that inhibitory regulation by SIRL-1 of monocytes or monocytederived cells plays a key role in skin homeostasis.

\section{Conclusion}

In summary, our study introduces with rs612529 an important genetic factor influencing the risk for skin inflammation. The identification of this link was made possible by defining an eQTL associated with these fluctuations from the whole blood data of our Chinese cohort and using the genotypes to mine pre-existing genetic association databases. A growing number of eQTL and GWAS data have been published already but only a fraction of the GWAS datasets are publicly accessible so far. Given the power the approach has to uncover new and unexpected links between genetically controlled phenotypes and disease manifestation, it appears to be mandatory that more of the "large data," including eQTL and GWAS, has to be compiled and curated in easily accessible repositories which are open for the general scientific community. In the case of SIRL-1 it not only helped to understand the role of the receptor in controlling immune cells, it also identified a novel candidate target for the treatment of AD. The identification of the as-yet unknown ligand(s) of SIRL-1 may thus open up new avenues to control or suppress the manifestation of the inflammatory skin disease.

\section{Additional files}

Additional file 1: Cohorts used in the study. The data for this study were collected from seven different cohorts. Four of these cohorts were established for the functional association studies (eQTL, FACS analysis, ROS assay, DNA methylation, and ChiP assay), while the three other cohorts were used for the genetic association studies with atopic dermatitis (AD). Size of the cohorts as well as ethnicity and appropriate reference are provided for each cohort. (PPTX $80 \mathrm{~kb}$ )

Additional file 2: Oligonucleotide sequences of probes used in EMSA experiments. The table lists the sequence of oligonucleotides used as radiolabeled probes or as cold competitors in EMSA. VSTM1 C and T probe sequences represent the two allelic variants of a 25 -bp region in the VSTM1 promoter containing the rs612529 SNP. Consensus and mutant oligonucleotides are based on consensus binding sites of the respective transcription factor. (PDF $51 \mathrm{~kb}$ )

Additional file 3: VSTM1 promoter sequence. The sequence of the region 411 bases upstream of the TSS and 282 bases downstream (untranslated region, Exon1, and part of Intron 1 of VSTM1 transcript) was obtained from UCSC genome browser (https://genome.ucsc.edu/). The location of rs612529 T/C (underlined) as well as of each CpG site is indicted (red bold font). The transcription start site is indicated by "TSS," the translational start by the start codon ATG. The transcribed region is indicated by small letters. Primers for bisulfite sequencing (F1/F2 and B1/ B2) as well as the primer pair used for rs612529 genotyping and ChiP analysis are indicated by empty arrows. (PDF $161 \mathrm{~kb}$ )

Additional file 4: Gating strategy for the flow cytometry analysis of SIRL-1 expression on whole blood samples. Whole blood samples stained with antibodies specific for CCR3, CD16, CD123, CD14, FCERla, and HLADR were counterstained with either anti-SIRL-1 or isotype-matched control antibody. Gates allowing the simultaneous analysis of the SIRL-1 staining on neutrophils, eosinophils, basophils, monocytes, mDC, pDC, B cells, and NK \& T cells are indicated. (PDF $210 \mathrm{~kb}$ )

Additional file 5: Gating strategy for monocytes expressing FcyRIII, FceRla, or FcaR. PBMCs were stained with antibodies specific for FcyRIII (CD16), FceRla, FcaR (CD89), and CD14 together with anti-SIRL-1, or isotype-matched control antibody. Monocytes were gated based on scatter properties (SSC-A and FSC-A) and live/dead-staining (AmCyan). Gates for FcyRIII+ monocytes, FceRla + monocytes, and FcaR+ monocytes are indicated in plots displaying the CD14 staining vs. the respective Fc-receptor. (PDF 297 kb)

Additional file 6: In silico prediction of transcription factors with allelespecific binding to rs612529. The web based prediction tools JASPAR (A) and TRANSFAC (B) were used to identify potential transcription factor binding sites in the VSTM1 promoter region affected by the allelic variations of rs612529 T/C. Only sites predicted with a binding score greater than 5.0 (JASPAR) and 0.9 (TRANSFAC) were selected for further analysis. The nucleotide underlined in red indicates the location of rs612529 within binding motif of the respective TF. Yellow shaded rows indicate transcription factors predicted to be highly specific for T allele.5. (PDF 55 kb)

Additional file 7: EMSA competition of PU.1 (ETS family) and YY1 consensus probes with rs612529 probes. EMSA experiments were performed with radiolabeled probes representing the consensus binding sequence of PU.1 and YY1. A EMSA competition experiment with consensus PU.1-probe. Nuclear extracts from primary monocytes were exposed to radiolabeled PU.1 consensus alone (lane 1) or together with excess of unlabeled PU.1 consensus probes (lane 2), PU.1 mutant probes (lane 3), or increasing amounts of rs612529 C (lanes 4-6) or T probes (lanes 7-9). The competitors were added to the binding reactions at increasing concentrations prior to the incubation with probe. B EMSA competition experiment with consensus YY1-probe. Radiolabeled YY1 probe was used alone (lane 1) or with excess of unlabeled YY1 probe (lane 2), YY1 mutant probe (lane 3), or increasing amounts of rs612529 C (lanes 4-7) or T probes (lanes 8-11). (PDF 243 kb)

Additional file 8: Model on the potentiating effect of a regulatory $\mathrm{CpG}$ SNP. A Conventional vs. regulatory CpG-SNPs. The methylation of CpG pairs results in the condensation of chromatin due to a tighter packaging of the histone-DNA complexes (open circle: non-methylated CpG, closed circle: methylated (pG). While a conventional CPG-SNP can assist this process by providing only one additional allele-dependent methylation site (middle panel), a regulatory CpG-SNP (such as rs612529) modulates the recruiting of $\mathrm{CpG}$-modifying enzymes (right panel). In the case of rs612529, this seems to be mediated by the allele-specific binding of PU.1 to the T allele of the CPG-SNP. In monocytes PU.1 was reported to bind the demethylase Tet-2. B Potentiating effect. Recruitment of Tet-2 mediates the chromatin-opening by facilitating the demethylation of all CpG pairs in the vicinity. The epigenetic effect is allele-specific (T allele) and direction of the direction probably cell-type-dependent. (PDF 415 kb)

\section{Acknowledgements}

The authors thank all apheresis blood donors from the Health Sciences Authority of Singapore for consenting to give blood for this study. We thank 
the volunteers and family members for participating in this study, as well as members of the Functional Genomics Laboratory at Department of Biological Sciences, National University of Singapore for their help with sample collection. We also thank Ivy Low, Nurhidaya Binte Shadan, and Seri Munriah Mustafah from the Flow Cytometry Facility. Esther Mok and Joni Chong from the Immunomonitoring platform at the Singapore Immunology Network for helping with the sample processing.

\section{Funding}

The study was supported by the Singapore Ministry of Education Academic Research Fund (R-154-000-404-112, R-154-000-553-112, R-154-000-565-112, R-154-000-630-112), National Medical Research Council (NMRC; Singapore, NMRC/1150/2008), and Singapore Immunology Network, Agency for Science, Technology and Research (A*STAR) Singapore (SlgN-06-006, SlgN-08-020, and SlgN-10-029).

\section{Availability of data and materials}

For the data relevant for the Caucasian eQTL study, the gene expression data are already available at www.ebi.ac.uk (E-MTAB-945), while the genotyping data are accessible at European Genome-Phenome Archive (EGA; EGAS00000000109) and available on request.

\section{Authors' contributions}

DK, KJP, and AKA performed conception and design; DK, KJP, GHAW, DH, ZL, and NY carried out the functional experiments while AKA, BL, RM, MP, DK, and KJP carried out analysis and interpretation; $J$, , GK, and FS, were involved in large-scale data generation while $\mathrm{J}, \mathrm{CKC}, \mathrm{SP}$, and DK were involved in the CHIP assay and interpretation of the epigenetics. ACA, JP, LDS, Al, JEAC, EBL, WDY, LJ, MT, MRJ, XZ, FTC, and FZ were involved in the association analysis and BPF, VN, and JK performed eQTL analysis in Caucasian cohorts. LM and OR were the lead investigators who led the study and facilitated the study design and execution. All authors were involved in drafting the manuscript for submission and provided feedback for subsequent revisions. All authors read and approved the final manuscript.

\section{Competing interests}

The authors declare that they have no competing interests.

\section{Consent for publication}

\section{Not applicable}

\section{Ethics approval and consent to participate}

All Singaporean cohorts are part of the Singapore Chinese Cohort Study, which was approved by the Institutional Review Board at the National University Hospital (IRB nos. NUS 07-023, NUS 10-445, and NUS 09-256) and are in compliance with the Helsinki declaration. Written informed consent was obtained from all volunteers prior to the collection of blood samples. All human apheresis cone blood used for the monocyte isolation was obtained from the Health Sciences Authority of Singapore and was approved by the National University of Singapore (IRB no. NUS-IRB 10-250) and is in compliance with the Helsinki declaration. Written informed consent was obtained for these donors and the samples were anonymized before they were accessible for research.

\section{Author details}

${ }^{1}$ Singapore Immunology Network (SlgN), A*STAR (Agency for Science, Technology and Research), 8A Biomedical Grove \#04-06, Singapore 138648, Republic of Singapore. ${ }^{2}$ Laboratory of Translational Immunology, Department of Immunology, University Medical Center Utrecht, P.O. box 85090, Utrecht $3508 \mathrm{AB}$, The Netherlands. ${ }^{3}$ Department of Epidemiology and Biostatistics, School of Public Health, Imperial College London, London, UK. ${ }^{4}$ Department of Environmental Health, National Institute for Health and Welfare, Kuopio, Finland. ${ }^{5}$ Institute of Dermatology and Department of Dermatology at No.1 Hospital, Anhui Medical University, Hefei, Anhui, China. ${ }^{6}$ Genome Institute of Singapore (GIS), Agency for Science, Technology and Research of Singapore (A*STAR), Singapore, Republic of Singapore. ${ }^{7}$ Wellcome Trust Centre for Human Genetics, Oxford, UK. ${ }^{8}$ Department of Oncology, Cancer and Haematology Centre, Churchill Hospital, Oxford, UK. Institute of Medical Biology (IMB), A*STAR (Agency for Science, Technology and Research), Singapore, Republic of Singapore. ${ }^{10}$ Department of Otolaryngology, National University of Singapore, Singapore, Republic of Singapore. "'Biological
Sciences, National University of Singapore, Singapore, Republic of Singapore. ${ }^{12}$ National Skin Center, Singapore, Republic of Singapore. ${ }^{13}$ Center for Life Course Epidemiology, Faculty of Medicine, University of Oulu, P.O. Box 5000, 90014 Oulu, Finland. ${ }^{14}$ Biocenter Oulu, University of Oulu, P.O. Box 5000, Aapistie 5A, 90014 Oulu, Finland. ${ }^{15}$ Unit of Primary Care, Oulu University Hospital, Kajaanintie 50, 90029 OYS, P.O. Box 20, 90220 Oulu, Finland.

${ }^{16}$ Department of Pathology, Singapore General Hospital, Singapore, Republic of Singapore. ${ }^{17}$ Institute of Molecular \& Cellular Biology (IMCB), Agency for Science, Technology and Research (A*STAR), Singapore 138648, Republic of Singapore. ${ }^{18}$ Department of Physiology, NUS Yong Loo Lin School of Medicine, National University of Singapore, Singapore, Republic of Singapore. ${ }^{19}$ Department of Biological Sciences, National University of Singapore, Singapore, Republic of Singapore.

Received: 10 August 2016 Accepted: 11 January 2017 Published online: 20 February 2017

\section{References}

1. Visscher PM, Brown MA, McCarthy MI, Yang J. Five years of GWAS discovery. Am J Hum Genet. 2012;90:7-24.

2. Manolio TA. Genomewide association studies and assessment of the risk of disease. N Engl J Med. 2010;363:166-76.

3. Chan SL, Jin S, Loh M, Brunham LR. Progress in understanding the genomic basis for adverse drug reactions: a comprehensive review and focus on the role of ethnicity. Pharmacogenomics. 2015;16:1161-78.

4. Welter D, MacArthur J, Morales J, Burdett T, Hall P, Junkins H, et al. The NHGRI GWAS Catalog, a curated resource of SNP-trait associations. Nucleic Acids Res. 2014;42:D1001-6.

5. Lappalainen T. Functional genomics bridges the gap between quantitative genetics and molecular biology. Genome Res. 2015;25:1427-31.

6. Pickrell JK, Marioni JC, Pai AA, Degner JF, Engelhardt BE, Nkadori E, et al. Understanding mechanisms underlying human gene expression variation with RNA sequencing. Nature. 2010;464:768-72.

7. Nica AC, Dermitzakis ET. Expression quantitative trait loci: present and future. Philos Trans R Soc Lond B Biol Sci. 2013;368:20120362.

8. Zhu Z, Zhang F, Hu H, Bakshi A, Robinson MR, Powell JE, et al. Integration of summary data from GWAS and eQTL studies predicts complex trait gene targets. Nat Genet. 2016;48:481-7.

9. Edwards SL, Beesley J, French JD, Dunning AM. Beyond GWASs: illuminating the dark road from association to function. Am J Hum Genet. 2013;93:779-97.

10. Huang Q. Genetic study of complex diseases in the post-GWAS era. J Genet Genomics. 2015;42:87-98.

11. Steevels TAM, Meyaard L. Immune inhibitory receptors: Essential regulators of phagocyte function. Eur J Immunol. 2011;41:575-87.

12. van Avondt K, Fritsch-Stork R, Derksen RHWM, Meyaard L. Ligation of signal inhibitory receptor on leukocytes-1 suppresses the release of neutrophil extracellular traps in systemic lupus erythematosus. PLoS ONE. 2012;8, e78459.

13. Vivier E, Daëron M. Immunoreceptor tyrosine-based inhibition motifs. Immunol Today. 1997;18:286-91.

14. Steevels TAM, Lebbink RJ, Westerlaken GHA, Coffer PJ, Meyaard L. Signal inhibitory receptor on leukocytes-1 is a novel functional inhibitory immune receptor expressed on human phagocytes. J Immunol. 2010;184:4741-8.

15. Ravetch JV, Lanier LL. Immune inhibitory receptors. Science. 2000;290:84-9.

16. Steevels TAM, van Avondt K, Westerlaken GHA, Stalpers F, Walk J, Bont L, et al. Signal inhibitory receptor on leukocytes-1 (SIRL-1) negatively regulates the oxidative burst in human phagocytes. Eur J Immunol. 2013;43:1297-308.

17. van Avondt $K$, van der Linden M, Naccache PH, Egan DA, Meyaard L. Signal inhibitory receptor on leukocytes-1 limits the formation of neutrophil extracellular traps, but preserves intracellular bacterial killing. J Immunol. 2016;196:3686-94.

18. Melchiotti R, Puan KJ, Andiappan AK, Poh TY, Starke M, Zhuang L, et al. Genetic analysis of an allergic rhinitis cohort reveals an intercellular epistasis between FAM134B and CD39. BMC Med Genet. 2014;15:73.

19. Fairfax BP, Makino S, Radhakrishnan J, Plant K, Leslie S, Dilthey A, et al. Genetics of gene expression in primary immune cells identifies cell type-specific master regulators and roles of HLA alleles. Nat Genet. 2012;44:502-10

20. Chen H, Common JEA, Haines RL, Balakrishnan A, Brown SJ, Goh CSM, et al. Wide spectrum of filaggrin-null mutations in atopic dermatitis highlights 
differences between Singaporean Chinese and European populations. Br J Dermatol. 2011;165:106.

21. Sun L-D, Xiao F-L, Li Y, Zhou W-M, Tang H-Y, Tang X-F, et al. Genome-wide association study identifies two new susceptibility loci for atopic dermatitis in the Chinese Han population. Nat Genet. 2011;43:690-4.

22. Rantakallio P. Groups at risk in low birth weight infants and perinatal mortality. Acta Paediatr Scand. 1969;193:1+.

23. Whitlock MC. Combining probability from independent tests: the weighted Z-method is superior to Fisher's approach. J Evol Biol. 2005;18:1368-73.

24. Lunyak W, Burgess R, Prefontaine GG, Nelson C, Sze S-H, Chenoweth J, et al. Corepressor-dependent silencing of chromosomal regions encoding neuronal genes. Science. 2002;298:1747-52.

25. Wu H, Caffo B, Jaffee HA, rizarry RA, Feinberg AP. Redefining CpG islands using hidden Markov models. Biostatistics. 2010;11:499-514.

26. Jaenisch R, Bird A. Epigenetic regulation of gene expression: how the genome integrates intrinsic and environmental signals. Nat Genet. 2003;33(Suppl):245-54.

27. Li T, Guo X, Wang W, Mo X, Wang P, Han W. V-set and transmembrane domain-containing 1 is silenced in human hematopoietic malignancy cell lines with promoter methylation and has inhibitory effects on cell growth. Mol Med Rep. 2014;11:1344-51.

28. Nakai K, Yoneda K, Kubota Y. Oxidative stress in allergic and irritant dermatitis: from basic research to clinical management. Recent Pat Inflamm Allergy Drug Discov. 2012;6:202-9.

29. Okayama Y. Oxidative stress in allergic and inflammatory skin diseases. Curr Drug Targets Inflamm Allergy. 2005:4:517-9.

30. Andiappan AK, Wang DY, Anantharaman R, Parate PN, Suri BK, Low HQ, et al. Genome-wide association study for atopy and allergic rhinitis in a Singapore Chinese population. PLoS ONE. 2011;6:e19719.

31. Scott EW, Simon MC, Anastasi J, Singh H. Requirement of transcription factor PU.1 in the development of multiple hematopoietic lineages. Science. 1994;265:1573-7.

32. Vangala RK, Heiss-Neumann MS, Rangatia JS, Singh SM, Schoch C, Tenen DG, et al. The myeloid master regulator transcription factor PU.1 is inactivated by AML1-ETO in t(8;21) myeloid leukemia. Blood. 2003;101:7.

33. Carotta S, Wu L, Nutt SL. Surprising new roles for PU.1 in the adaptive immune response. Immunol Rev. 2010;238:63-75.

34. van Riel B, Rosenbauer F. Epigenetic control of hematopoiesis: the PU.1 chromatin connection. Biol Chem. 2014;395:1265-74.

35. la Rica De L, Rodríguez-Ubreva J, García M, Islam ABMMK, Urquiza JM, Hernando H, et al. PU.1 target genes undergo Tet2-coupled demethylation and DNMT3b-mediated methylation in monocyte-to-osteoclast differentiation. Genome Biol. 2012;14:R99-9.

36. Deaton AM, Bird A. CpG islands and the regulation of transcription. Genes Dev. 2011;25:1010-22.

37. Klose RJ, Bird AP. Genomic DNA methylation: the mark and its mediators. Trends Biochem Sci. 2006;31:89-97.

38. Zhi D, Aslibekyan S, Irvin MR, Claas SA, Borecki IB, Ordovas JM, et al. SNPS located at $\mathrm{CpG}$ sites modulate genome-epigenome interaction. Epigenetics. 2013:8:802-6.

39. Ziller MJ, Gu H, Müller F, Donaghey J, Tsai LT-Y, Kohlbacher O, et al. Charting a dynamic DNA methylation landscape of the human genome. Nature. 2013;500:477-81.

40. Shoemaker R, Deng J, Wang W, Zhang K. Allele-specific methylation is prevalent and is contributed by $\mathrm{CpG}-\mathrm{SNPS}$ in the human genome. Genome Res. 2010;20:883-9.

41. Guttman-Yassky E, Nograles KE, Krueger JG. Contrasting pathogenesis of atopic dermatitis and psoriasis-part I: clinical and pathologic concepts. J Allergy Clin Immunol. 2011;127:1110-8.

42. Leung DYM. New insights into atopic dermatitis: role of skin barrier and immune dysregulation. Allergol Int. 2013:62:151-61.

43. Illi S, von Mutius E, Lau S, Nickel R, Grüber C, Niggemann B, et al. The natural course of atopic dermatitis from birth to age 7 years and the association with asthma. J Allergy Clin Immunol. 2004;113:925-31.

44. Aghamohammadi A, Cheraghi T, Gharagozlou M, Movahedi M, Rezaei N, Yeganeh $\mathrm{M}$, et al. IgA deficiency: correlation between clinical and immunological phenotypes. J Clin Immunol. 2008;29:130-6.

\section{Submit your next manuscript to BioMed Central and we will help you at every step:}

- We accept pre-submission inquiries

- Our selector tool helps you to find the most relevant journal

- We provide round the clock customer support

- Convenient online submission

- Thorough peer review

- Inclusion in PubMed and all major indexing services

- Maximum visibility for your research

Submit your manuscript at www.biomedcentral.com/submit
Biomed Central 